PSICOLOGÍA

IBEROAMERICANA
Psicología Iberoamericana ISSN: 1405-0943

revista.psicologia@ibero.mx

Universidad Iberoamericana, Ciudad de México México

\title{
EI COVID-19 y la salud mental: ¿cuáles son las consecuencias?
}

Gordon, Sarah Frances

EI COVID-19 y la salud mental: ¿cuáles son las consecuencias?

Psicología Iberoamericana, vol. 28, núm. 1, 2020

Universidad Iberoamericana, Ciudad de México, México

Disponible en: http://www.redalyc.org/articulo.oa?id=133963198003 


\title{
Editorial
}

\section{El COVID-19 y la salud mental: ¿cuáles son las consecuencias?}

\author{
Sarah Frances Gordon sarah.gordon@ibero.mx \\ Universidad Iberoamericana, Ciudad de México, México \\ http://orcid.org/0000-0001-5131-8519
}

Psicología Iberoamericana, vol. 28, núm. 1,2020

Universidad Iberoamericana, Ciudad de México, México

Redalyc: http://www.redalyc.org/ articulo.oa?id=133963198003
La pandemia del COVID-19, que se cree se originó en 'un mercado mojado $^{\text {[1] }}$ en Wuhan, provincia de Hubei, China a finales de 2019, se ha extendido por todo el mundo (Xiang et al., 2020). El 28 de febrero de 2020, México confirmó sus primeros casos de COVID-19 en dos hombres que habían viajado recientemente a Italia (Perla \& Morales, 2020). Desde el 8 de mayo de 2020, como se indica en el sitio web del gobierno, México tiene cerca de 30 mil casos positivos y alrededor de 3 mil muertes confirmadas por COVID-19 (COVID -19 Tablero México-conacyt-CentroGeo-GeoInt-DataLab', 2020 ${ }^{[2]}$. Se espera que estas cifras aumenten y, para combatir la pandemia, el gobierno mexicano ha cerrado sectores de la economía y pedido a la población que se mantenga en casa para ayudar a "aplanar la curva" y detener la propagación de nuevas infecciones. A nivel mundial, existen casi cuatro millones de casos confirmados en 187 países y aproximadamente 270 mil personas han fallecido a causa del COVID-19 ${ }^{[3]}$ (BBC, 2020).

A medida que los casos provocados por este virus aumentan, tanto a nivel internacional como en México, la Organización Mundial de la Salud (OMS) y otras autoridades sanitarias de todo el mundo han recomendado medidas como el distanciamiento social y las cuarentenas para combatir la infección (Shah et al., 2020). Sin embargo, dichas medidas, que representan estrés por sí solas, también implican que el gobierno cierre secciones de la economía en interés de la salud pública, lo que lleva a que muchas personas no puedan trabajar y generar ingresos. Esto conduce a la ansiedad y el miedo entre la población general, que también se combina con las crecientes preocupaciones que se tienen por el bienestar y la salud de los hijos, de los familiares de edad avanzada, y de aquellos en condiciones de alto riesgo y con sistemas inmunodeprimidos. El sentimiento palpable de miedo y ansiedad que todos experimentamos ahora, hace que la conexión entre los problemas de la salud mental y el coronavirus parezca obvia.

Hay una cantidad creciente de literatura académica sobre la relación entre los riesgos de salud mental y el COVID-19. Gran parte de ella proviene de Asia, principalmente de China (Kang, Li, et al., 2020; Kang, 
Ma, et al., 2020; Lai et al., 2020; Li et al., 2020; Xiang et al., 2020), Japón ( Shigemura, Ursano, Morganstein, Kurosawa, \& Benedek, 2020) y Corea del Sur (Park \& Park, 2020), pero también hay otras contribuciones de América del Sur (Lima et al., 2020; Torales, O’Higgins, Castaldelli-Maia, \& Ventriglio, 2020), Europa (Fiorillo \& Gorwood, 2020; Wind, Rijkeboer, Andersson, \& Riper, 2020), e India (Shah et al., 2020). Todos estos estudios ponen de relieve los riesgos para la salud mental a los que se enfrentan los trabajadores de la salud, en particular el personal médico, así como la afectación al conjunto de la población, ya que todos estamos siendo sometidos a cuarentenas masivas y cierres del transporte público.

Las investigaciones muestran que las cuarentenas domiciliarias están relacionadas con el estrés psicológico y representan un peligro para la salud mental (Shah et al., 2020). Barbisch, Koenig y Shih (2015) encontraron en su estudio sobre el papel de la cuarentena en la lucha contra enfermedades infecciosas como el SARS y el Ébola, que poner a las poblaciones bajo aislamiento puede conducir a severos problemas psicológicos, agravados por el miedo y la incertidumbre de las enfermedades. Además, el confinamiento de individuos en espacios pequeños durante períodos prolongados, bajo la amenaza de contraer una enfermedad, puede conducir a la histeria masiva y a la desintegración de la salud mental (Barbisch et al., 2015). Esto se observó en el personal del hospital que estaba en cuarentena al tratar el SARS en Taipéi, Taiwán, y se encontró que este tipo de confinamiento llevó al personal a extremos como el suicidio.

El aumento de la soledad y la reducción de las interacciones sociales también son factores de riesgo de trastornos psicológicos como la esquizofrenia y la depresión mayor (Fiorillo \& Gorwood, 2020). HoltLunstad, Smith, Baker, Harris y Stephenson (2015) encontraron, en su revisión meta-analítica de la literatura sobre el tema de la soledad y el aislamiento social, que éste conduce a una mayor mortalidad y representa un mayor riesgo para la salud individual que la obesidad. Holt-Lunstad et al. (2015) argumentan que, a la luz de esta investigación, el aislamiento social debe ser presentado como un tema importante de salud pública. Esto es ciertamente indudable en los tiempos del COVID-19, donde las cuarentenas son comunes y se fomenta el distanciamiento social. Las implicaciones psicológicas de instruir a las personas a permanecer en cuarentena doméstica combinadas con las preocupaciones sobre su salud y la de su familia, así como la incertidumbre sobre la situación futura y económica, pueden generar o exacerbar sentimientos de ansiedad, depresión y miedo (Fiorillo \& Gorwood, 2020). Teniendo todo esto en cuenta, debería haber un cambio hacia el énfasis en la salud mental a nivel mundial y local en medio de esta pandemia.

Como psicólogos y trabajadores de la salud mental, debemos prepararnos para este nuevo desafío a medida que se da mayor importancia a la salud mental. Las prioridades de la población están cambiando a medida que la pandemia representa un factor de estrés relativamente nuevo en sus vidas, y el número de personas que necesitarán 
intervención psicológica se elevará en las próximas semanas y meses (Fiorillo \& Gorwood, 2020). Debemos esperar un aumento de los problemas de salud mental y los trastornos por el abuso de sustancias en la población, ya que los factores de estrés extremos como la pérdida de ingresos, el miedo, las enfermedades en la familia, el aislamiento social y la ansiedad, pueden exacerbar o generar condiciones psiquiátricas (Fiorillo \& Gorwood, 2020).

Shah et al. (2020, p. 4) sugieren que para evitar los efectos en la salud mental derivados de esta enfermedad, "las personas deben evitar la exposición excesiva a las coberturas del COVID-19 en los medios de comunicación, mantener una dieta saludable y un estilo de vida positivo, y extender la mano en busca de consuelo". Las personas deben tratar de limitar las fuentes de estrés y confiar sólo en una cantidad limitada de información ofrecida por los canales oficiales (Fiorillo \& Gorwood, 2020). Aumentar la comunicación con la familia, amigos y seres queridos, a través de video-chats y llamadas telefónicas, es importante para ayudar a romper las consecuencias negativas asociadas con el aislamiento social (Fiorillo \& Gorwood, 2020). Desarrollar una rutina diaria también es útil, ya que comunica una sensación de normalidad al cuerpo. La OMS recomienda una combinación de actividad física regular, alimentación saludable, y una mayor conciencia de su salud mental para combatir el estrés por el COVID -19 (OMS, 2020). Tratar de enfocarse en los beneficios de esta etapa también puede ser útil, así como estar agradecido por el mayor tiempo de calidad con la familia, hijos y pareja (Fiorillo \& Gorwood, 2020). Además, es importante pedir ayuda profesional si usted siente que no está haciendo frente a la situación y buscar servicios de salud mental.

Como profesionales e investigadores de la salud mental, necesitamos trazar un camino hacia adelante y aceptar que el mundo se ha transformado, y por lo tanto tenemos que cambiar con él. Tenemos que entender que cada vez más personas corren un mayor riesgo de desarrollar problemas psiquiátricos debido a la introducción de nuevos factores de estrés; así mismo debemos estar preparados para hacer frente a esto. Lo más importante es abordar el estigma asociado a los problemas de salud mental que existe en México y fomentar discusiones abiertas sobre el tema en nuestras comunidades, así como una mayor conciencia respecto al constante monitoreo de la salud mental.

\section{Notas al pie}

[1] Un mercado mojado es un mercado que vende carne fresca, pescado y otros productos perecederos a precios accesibles. No debe confundirse con los mercados de vida silvestre en China y Asia, que comercian con animales vivos y exóticos (Beech, 2020; Yu, 2020). El agua se lanza regularmente sobre los productos para mantenerlo fresco y el hielo derretido se utiliza de manera regular como una técnica de enfriamiento para los peces (Beech, 2020; Yu, 2020). De ahí proviene el término "mercado mojado". 
[2] Estas estadísticas han sido puestas en disputa, ya que muchos trabajadores del sector salud y funcionarios de la Ciudad de México sospechan que el gobierno federal no está reportando cientos o incluso miles de muertes derivadas del COVID-19, así como casos positivos en México. (Azam, 2020).

[3] Estas cifras pueden haber aumentado desde el momento en que este artículo se haya escrito.

\section{Referencias}

Azam, A. (2020, May 8). Hidden toll: Mexico ignores wave of Coronavirus deaths in capital. New York Times. Recuperado https://www.nytimes.co $\mathrm{m} / 2020 / 05 / 08 /$ world/americas/mexico-coronavirus-count.html

Barbisch, D., Koenig, K. L., \& Shih, F.-Y. (2015). Is there a case for quarantine? Perspectives from sars to Ebola. Disaster Medicine and Public Health Preparedness, 9(5), 1-13. https://doi.org/10.1017/dmp.2015.38

BBC. (2020, May 8). Coronavirus pandemic: Tracking the global outbreak. BBC News. Recuperado de https://www.bbc.com/news/world-5123510 5

Beech, P. (2020). What are China's wet markets? World Economic Forum. Recuperado de https://www.weforum.org/agenda/2020/04/china-wetmarkets-covid19-coronavirus-explained/

Covid-19 Tablero México-conacyt-CentroGeo-GeoInt-DataLab. (2020). Recuperado 8 May 2020 de https://coronavirus.gob.mx/datos/

Fiorillo, A., \& Gorwood, P. (2020, April 1). The consequences of the COVID-19 pandemic on mental health and implications for clinical practice. European Psychiatry: The Journal of the Association of European Psychiatrists. nlm (Medline). https://doi.org/10.1192/j.eurpsy.2020.35

Holt-Lunstad, J., Smith, T. B., Baker, M., Harris, T., \& Stephenson, D. (2015). Loneliness and social isolation as risk factors for mortality. Perspectives on Psychological Science, 10(2), 227-237. https://doi.org/10.1177/1745691 614568352

Kang, L., Li, Y., Hu, S., Chen, M., Yang, C., Yang, B. X., ... Liu, Z. (2020). The mental health of medical workers in Wuhan, China dealing with the 2019 novel coronavirus. The Lancet Psychiatry, 7(3), e14. https://doi.org/10.1 016/S2215-0366(20)30047-X

Kang, L., Ma, S., Chen, M., Yang, J., Wang, Y., Li, R., ... Liu, Z. (2020). Impact on mental health and perceptions of psychological care among medical and nursing staff in Wuhan during the 2019 novel coronavirus disease outbreak: A cross-sectional study. Brain, Behavior, and Immunity. https: //doi.org/10.1016/j.bbi.2020.03.028

Lai, J., Ma, S., Wang, Y., Cai, Z., Hu, J., Wei, N., ... Hu, S. (2020). Associated with mental health outcomes among health care workers. JAMA Network Open, 3(3), 203976. https://doi.org/10.1001/jamanetworkopen.2020.39 76

Li, W., Yang, Y., Liu, Z. H., Zhao, Y. J., Zhang, Q., Zhang, L., ... Xiang, Y. T. (2020). Progression of mental health services during the COVID-19 outbreak in China. International Journal of Biological Sciences, 16(10), 1732-1738. https://doi.org/10.7150/ijbs.45120 
Lima, C. K. T., Carvalho, P. M. de M., Lima, I. de A. A. S., Nunes, J. V. A. de O., Saraiva, J. S., de Souza, R. I., ... Neto, M. L. R. (2020, May 1). The emotional impact of Coronavirus 2019-nCoV (new Coronavirus disease). Psychiatry Research. Elsevier Ireland Ltd. https://doi.org/10.1016/j.psychres.2020.1 12915

OMS (2020). \#HealthyAtHome. Recuperado 11 May 2020 de https://www.w ho.int/news-room/campaigns/connecting-the-world-to-combat-corona virus/healthyathome?gclid=CjwKCAjw7-P1BRA2EiwAXoPWA3yT4 bLmAA-tQXWwfujNEsHi8oM9on6ZnNDae4DQ9CcLn1iWxOrlOx oC9S8QAvD_BwE

Park, S. C., \& Park, Y. C. (2020, February 1). Mental health care measures in response to the 2019 novel coronavirus outbreak in Korea. Psychiatry Investigation. Korean Neuropsychiatric Association. https://doi.org/10.3 0773/pi.2020.0058

Perla, M., \& Morales, A. (2020, February 28). Se confirma primer caso de COVID-19 en México. El Universal. Recuperado de https://www.eluniversal.com.mx/nacion/sociedad/coronavirus-en-m exico-confirman-primer-caso

Shah, K., Kamrai, D., Mekala, H., Mann, B., Desai, K., \& Patel, R. S. (2020). Focus on mental health during the coronavirus (COVID-19) pandemic: Applying learnings from the past outbreaks. Cureus, 12(3), e7405. https: //doi.org/10.7759/cureus.7405

Shigemura, J., Ursano, R. J., Morganstein, J. C., Kurosawa, M., \& Benedek, D. M. (2020). Public responses to the novel 2019 coronavirus (2019-nCoV) in Japan: Mental health consequences and target populations. Psychiatry and Clinical Neurosciences, 74(4), 281-282. https://doi.org/10.1111/pc n. 12988

Torales, J., O’Higgins, M., Castaldelli-Maia, J. M., \& Ventriglio, A. (2020). The outbreak of COVID-19 coronavirus and its impact on global mental health. International Journal of Social Psychiatry. https://doi.org/10.1177 /0020764020915212

Wind, T. R., Rijkeboer, M., Andersson, G., \& Riper, H. (2020). The COVID-19 pandemic: The 'black swan' for mental health care and a turning point for e-health. Internet Interventions, 20, 100317. https://doi.org/10.1016/j.in vent.2020.100317

Xiang, Y. T., Yang, Y., Li, W., Zhang, L., Zhang, Q., Cheung, T., \& Ng, C. H. (2020). Timely mental health care for the 2019 novel coronavirus outbreak is urgently needed. The Lancet Psychiatry, 7(3), 228-229. https: //doi.org/10.1016/S2215-0366(20)30046-8

Yu, V. (2020, April 16). What is a wet market? The Guardian. Retrieved from https://www.theguardian.com/global-development/2020/apr/16/ what-is-a-wet-market-coronavirus 